Journal of Computer Science 7 (7): 958-961, 2011

ISSN 1549-3636

(C) 2011 Science Publications

\title{
Content Based Image Retrieval using Novel Gaussian Fuzzy Feed Forward-Neural Network
}

\author{
${ }^{1}$ C. Ramesh Babu Durai and ${ }^{2}$ V. Duraisamy \\ ${ }^{1}$ Anna University of Technology Coimbatore \\ Coimbatore, 641 047, Tamil Nadu, India \\ ${ }^{2}$ Hindusthan College of Engineering and Technology, \\ Coimbatore-641 032, Tamil Nadu, India
}

\begin{abstract}
Problem statement: With extensive digitization of images, diagrams and paintings, traditional keyword based search has been found to be inefficient for retrieval of the required data. Content-Based Image Retrieval (CBIR) system responds to image queries as input and relies on image content, using techniques from computer vision and image processing to interpret and understand it, while using techniques from information retrieval and databases to rapidly locate and retrieve images suiting an input query. CBIR finds extensive applications in the field of medicine as it assists a doctor to make better decisions by referring the CBIR system and gain confidence. Approach: Various methods have been proposed for CBIR using image low level image features like histogram, color layout, texture and analysis of the image in the frequency domain. Similarly various classification algorithms like Naïve Bayes classifier, Support Vector Machine, Decision tree induction algorithms and Neural Network based classifiers have been studied extensively. We proposed to extract features from an image using Discrete Cosine Transform, extract relevant features using information gain and Gaussian Fuzzy Feed Forward Neural Network algorithm for classification. Results and Conclusion: We apply our proposed procedure to 180 brain MRI images of which 72 images were used for testing and the remaining for training. The classification accuracy obtained was $95.83 \%$ for a three class problem. This research focused on a narrow search, where further investigation is needed to evaluate larger classes.
\end{abstract}

Key words: Data mining, Content-Based Image Retrieval (CBIR), image retrieval, discrete cosine transform, information gain, neural network, Gaussian fuzzy feed forward, pathological image, classification algorithm

\section{INTRODUCTION}

Future medical information systems will play a very important role in clinical decision making process by providing similar pathological conditions in a medical image and thus help the physician view the significant images to make a better decision. CBIR has been effectively used to retrieve images from databases based on the query input which can either be an anatomical region or pathological image.

Traditional image retrieval is based on text query and this approach has been extended to image retrieval by using an query image as the input. Typical content based image retrieval systems rely on low level visual features such as texture, shape, color and spatial relationships addresses the detailed perceptual aspects and high level semantic features such as text descriptors addresses the conceptual aspect of the image.

\section{MATERIALS AND METHODS}

Color has been extensively used in CBIR systems due to its simplicity. Various perception of color spaces have been proposed including color histogram (Gavrielides et al., 2006) correlograms and anglograms.

Texture is the innate property of an object made of repetitive structure of a particular element in relative spatial positions. Some of the characteristics of texture are contrast, regularity, coarseness, directionality and roughness. Texture feature extraction has been widely done using Gabor filters (Greenspan et al., 2007). Texture features are compared based on the dissimilarity between vector features using Euclidean of Mahalanobis distance (Helmy et al., 2010).

Another popular low level feature is the use of shapes in an image to distinguish it. Shapes can either

Corresponding Author: C. Ramesh Babu Durai, Anna University of Technology Coimbatore Coimbatore, 641 047,

Tamil Nadu, India 
be the boundary of the image or can be based on region of interest. Shapes derived from subpart of the object are called as local shapes whereas if shapes are derived from the entire object, they are called as global shapes.

For an effective CBIR system a combination of the above mentioned features are used. Of the numerous methods proposed for feature extraction, frequency representation of the image offer high levels of invariance to noise and is preferred by many researchers in the area of content based image retrieval (Shareha et al., 2009)

Suhasini et al. (2008) proposed a graph based segmentation approach to distinguish low variability image regions and high variability image regions for feature extraction from segments.

Classifiers based on Bayesian network (Cox et al., 2000; De Ves et al., 2006), Self organizing maps (Koskela et al., 2004), Support vector machine (Zhou and Huang, 2001; Tong and Chang, 2001) and Regression models (Leon et al., 2007) have been proposed with varying classification accuracy.

Ghrare et al. (2009) proposed lossless coding for the image retrieval problem by using lossless compression with high accuracy.

Treating CBIR as a classification problem leads to creation of features with very high dimensionality. Overcoming the high dimensionality without losing the image retrieval ability is a challenge in research. In this study we propose to extract feature vectors from MRI medical images using the Discrete Cosine Transform, extract sub features using the information gain of each attribute and propose a novel neural network classifier called Gaussian Fuzzy Feed Forward Neural Network (GFFF-NN) in which we introduce a fuzzy component in the hidden layer. The next sections of this study discuss the proposed model, experimental setup and results obtained. The final section concludes our study with a discussion of the obtained result.

\section{RESULTS AND DISCUSSION}

Proposed Gaussian fuzzy feed forward neural network: Numerous neural network models have been proposed over the last 5 decades and most of the models are based on the Hopfield network (Hertz et al., 1991) and perceptron (Haykin, 1998). Based on the type of feedback Neural networks can be classified as feedforward and feedback models. In this study we concentrate on feedforward networks with supervised learning. In supervised learning the training set consists of input output pairs and there may be an arbitrary association between some inputs and outputs. These pairs may correspond to a mapping function along with the classes to which they belong. The desired output is obtained by reducing the error between the network output and the actual output.
The training set of size $\mathrm{p}$ can be represented as $T_{P}$

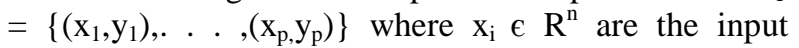
vectors of dimension $n$ and $y_{i} \in R^{m}$ are the output vectors of dimension $m$ and $R$ represents the set of real numbers. Let $f_{w}$ represent the function with weight $w$ for the neural network. Supervised learning adjusts the weight such that:

$$
\mathrm{f}_{\mathrm{w}}\left(\mathrm{x}_{\mathrm{i}}\right)=\mathrm{y} ; \forall\left(\mathrm{x}_{\mathrm{i}}, \mathrm{y}_{\mathrm{i}}\right) \in \mathrm{T}_{\mathrm{p}}
$$

When a Neural network is trained and is tested on new samples its output may be correct to a certain extent. But generalization does not help partial correctness. The network considers whether a sample gives the desired output or not. Partial correctness can be solved using a fuzzy approach.

The Gaussian Fuzzy Feed Forward Neural Network (GFFF-NN) proposed in this study uses the criteria specified in Table 1.

The hidden layer consists of transfer function is given by:

$f\left(x_{i}\right)=f\left(x_{i}, w_{i}\right)$

Being a Gaussian function the equation for $\mathrm{f}\left(\mathrm{x}_{\mathrm{i}}, \mathrm{w}_{\mathrm{i}}\right)$ is given by:

$f\left(x_{i}, w_{i}\right)=\exp \left[-\beta_{i}\left(x_{i}+w_{i}\right)^{2}\right]$

The Gaussian transfer function responds significantly to a local area of the input space and is used for local function approximation.

The proposed Gaussian Fuzzy membership function is given by:

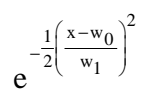

where, $\mathrm{w}_{1}$ and $\mathrm{w}_{2}$ are the stored weight parameters.

Table 1: Architecture of the proposed neural network

\begin{tabular}{ll}
\hline Input Neuron & 200 \\
Output Neuron & 3 \\
Number of hidden layer & 1 \\
Number of processing elements & 4 \\
Transfer function of hidden layer & Fuzzy Gaussian \\
Learning Rule of hidden layer & Momentum \\
Step size & 0.1 \\
Momentum & 0.7 \\
Transfer function of output layer & TanH \\
Learning Rule of output layer & Momentum \\
Step size & 0.1 \\
Momentum & 0.7 \\
Maximum Epoch & 500 \\
\hline
\end{tabular}


To validate the classification algorithm we used 180 MRI images. Sample images are shown in Fig. 1. The co efficient were extracted using Discrete Cosine Transform given by:

$$
\begin{aligned}
& F(u, v)=\left(\frac{2}{N}\right)^{\frac{1}{2}}\left(\frac{2}{M}\right)^{\frac{1}{2}} \sum_{i=0}^{N-1} \sum_{j=0}^{M-1} \Lambda(i) \cdot \Lambda(j) . \\
& \cos \left[\frac{\pi \cdot u}{2 \cdot N}(2 i+1)\right] \cos \left[\frac{\pi \cdot v}{2 \cdot M}(2 j+1)\right] . f(i, j)
\end{aligned}
$$

The co efficient so obtained was mapped to the class and the information gain was computed and ranked based on the class label. The information gain used in the calculation is given by.

Let Attr be the set of all attributes and Ex the set of all training examples, value(x,a) with $x \in E x$ defines the value of a specific example $x$ for attribute $x \in A t t r, H$ specifies the entropy and $|\mathrm{x}|$ is the number of elements in the set $x$. The information gain for an attribute $\mathrm{a} \in$ Attr is defined as follows:

$$
\begin{aligned}
& \mathrm{IG}(\mathrm{Ex}, \mathrm{a})=\mathrm{H}(\mathrm{Ex})-\sum_{\mathrm{v} \in \text { values(a) }} \frac{|\{\mathrm{x} \in \mathrm{Ex} \mid \operatorname{value}(\mathrm{x}, \mathrm{a})=\mathrm{v}\}|}{|\operatorname{Ex}|} . \\
& \mathrm{H}(\{\mathrm{x} \in \mathrm{Ex} \mid \operatorname{value}(\mathrm{x}, \mathrm{a})=\mathrm{v}\})
\end{aligned}
$$

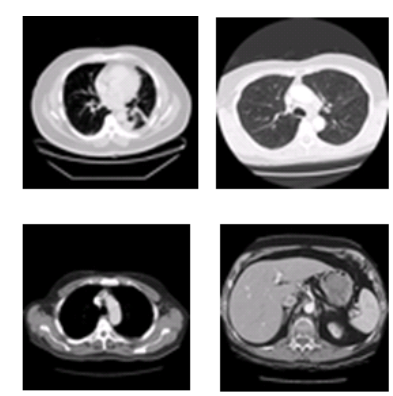

Fig. 1: Sample images used

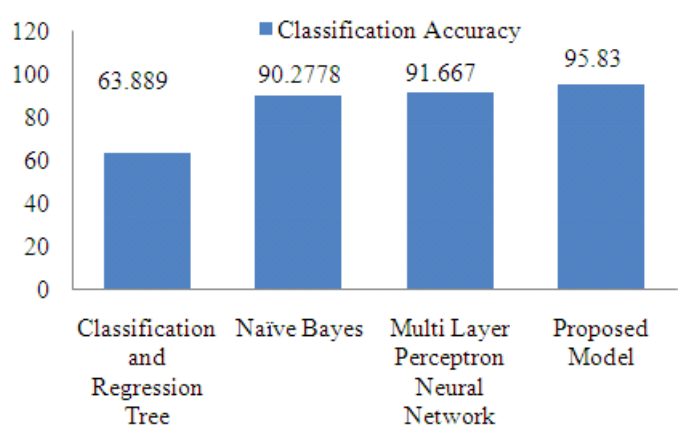

Fig. 2: Classification accuracy of various classifiers and our proposed classifier
The obtained information gain was ranked and the first 200 ranks were selected as the attributes for the proposed neural network classification algorithm.

The results obtained showed a classification accuracy of $95.83 \%$. The proposed model was benchmarked with other models including Naïve Bayes, CART and Multilayer perceptron Neural Network. The results obtained are shown in Fig. 2.

\section{CONCLUSION}

In this study we proposed a novel neural network classification algorithm for MRI medical image classification. Image feature was obtained using Discrete Cosine Transform and information gain was computed on the obtained co efficient. The information gained was ranked and the first 200 parameters were selected as attributes for our proposed algorithm. The Classification algorithm developed was benchmarked with other classifiers and its performance is better than the other classifiers

\section{REFERENCES}

Cox, I.J., M.L. Miller, T.P. Minka, T.V. Papathomas and P.N. Yianilos. 2000. The Bayesian image retrieval system, pichunter: Theory, implementation and psychophysical experiments. IEEE Trans. Image Process., 9: 20-37. DOI: $10.1109 / 83.817596$

De Ves, E, J. Domingo, G. Ayala and P. Zuccarello. 2006. A novel Bayesian framework for relevance feedback in image content-based retrieval systems. Patt. Recog., 39: 1622-1632. DOI: 10.1016/J.PATCOG.2006.01.006

Gavrielides, M.A, E. Sikudova and I. Pitas, 2006. Color-based descriptors for image fingerprinting. IEEE Trans. Multimedia, 8: 740-748. DOI: 10.1109/TMM.2006.876290

Ghrare, S.E., M.A.M. Ali, K. Jumari and M. Ismail, 2009. An efficient low complexity lossless coding algorithm for medical images. Am. J. Applied Sci., 6: 1502-1508. DOI: 10.3844/ajassp.2009.1502.1508

Greenspan, H. and A.T. Pinhas, 2007. Medical image categorization and retrieval for PACS using the GMM-KL framework. IEEE Trans. Inform. Technol. Biomed., 11: 190-202. DOI: 10.1109/TITB.2006.874191

Haykin, S., 1998. Neural Networks: A Comprehensive Foundation. 2nd Edn., Prentice Hall, ISBN-10: 0132733501, pp: 842. 
Helmy A.K. and G.S. El-Taweel, 2010. Neural network change detection model for satellite images using textural and spectral characteristics. Am. J. Eng. Applied Sci., 3: 604-610. DOI: 10.3844/ajeassp.2010.604.610

Hertz, J.A., A.S. Krogh and R.G. Palmer, 1991. Introduction to the Theory of Neural Computation. Westview Press, ISBN-10: 0201515601, pp: 350.

Koskela M., J. Laaksonen and E. Oja, 2004. Use of image subset features in image retrieval with selforganizing maps. Lecture Notes Comput. Sci., 508516.

http://direct.bl.uk/bld/PlaceOrder.do?UIN=153246 486\&ETOC $=\mathrm{RN} \&$ from $=$ searchengine

Leon, T, P. Zuccarello, G. Ayala, E.D. Ves and J. Domingo, 2007. Applying logistic regression to relevance feedback in image retrieval systems. Patt. Recog., 40: 2621-2632. DOI: 10.1016/J.PATCOG.2007.02.002

Shareha, A.A.A., M. Rajeswari and D. Ramachandram, 2009. Multimodal integration (image and text) using ontology alignment. Am. J. Applied Sci., 6: 1217-1224. DOI: 10.3844/ajassp.2009.1217.1224
Suhasini, P.S., K.S.R. Krishna and I.V.M. Krishna, 2008. Graph based segmentation in content based image retrieval. J. Comput. Sci., 4: 699-705. http://www.scipub.org/fulltext/jcs/jcs48699705.pdf

Tong, S. and E. Chang, 2001. Support vector machine active learning for image retrieval. Proceedings of the 9th ACM International Conference on Multimedia, Sept. 30-Oct. 05, Ottawa, ON, Canada, pp: 107-118. DOI: 10.1145/500141.500159

Zhou, X.S. and T.S. Huang, 2001. Small sample learning during multimedia retrieval using biasmap. Proceedings of the IEEE Conference on Computer Vision and Pattern Recognition, Dec. 08-14, Kauai, Hawaii, pp: 11-17. http://www.computer.org/portal/web/csdl/doi/10.11 09/CVPR.2001.990450 